\title{
Competencias en el profesorado universitario derivadas del impacto de la COVID-19
}

\author{
Carlos Martínez Mollineda ${ }^{1}$ \\ Raxiel Martínez Pedraza ${ }^{1}$ (it) \\ Dainury Vázquez Coll ${ }^{1}$ (D) \\ Universidad Central "Marta Abreu" de Las Villas, Cuba
}

Resumen. La pandemia COVID-19 ha puesto a prueba las Instituciones de Educación Superior del mundo. Cierre parcial o total de las aulas, adaptaciones curriculares y el proceso de enseñanzaaprendizaje detenido son algunas de las consecuencias negativas de la pandemia. El objetivo de este artículo es definir cuáles son las competencias desarrolladas por el docente universitario para enfrentar metodológicamente la docencia en condiciones de la COVID-19. En la investigación participan 12 docentes de la Universidad Central "Marta Abreu" de Las Villas. La investigación, con enfoque mixto, no experimental y carácter descriptivo, se realiza en dos etapas. La primera, determina las competencias claves del docente universitario para enfrentar metodológicamente la docencia en condiciones de la COVID-19. La segunda, describe el estado actual de estas competencias claves en el profesorado universitario. En la investigación se aplican tres instrumentos (guía para la revisión bibliográfica, composición y cuestionario). Los resultados muestran la necesidad de continuar diagnosticando, en la práctica, la efectividad del modelo de educación a distancia y continuar desarrollando las competencias claves para enfrentar metodológicamente la docencia en condiciones de la COVID-19. Se propone ampliar la investigación a un universo mayor de docentes para diagnosticar e influir en las competencias que aún no han sido lo suficientemente desarrolladas.

Palabras clave: competencias docentes; enseñanza; aprendizaje; COVID-19; educación.

Competências do corpo docente universitário derivadas do impacto do COVID-19

Resumo. A pandemia do COVID-19 colocou à prova as Instituições de Ensino Superior do mundo. O fechamento parcial ou total das salas de aula, as adaptações curriculares e o processo de ensinoaprendizagem bloqueado são algumas das consequências negativas da pandemia. O objetivo deste artigo é definir as competências desenvolvidas pelo docente universitário para enfrentar metodologicamente o ensino nas condições do COVID-19. Participam da pesquisa 12 docentes da Universidade Central "Marta Abreu" de Las Villas. A pesquisa, com abordagem mista, não experimental e descritiva, é realizada em duas etapas. A primeira determina as competências-chave do docente universitário para enfrentar metodologicamente o ensino nas condições do COVID-19. A segunda descreve o estado atual dessas competências-chave no corpo docente universitário. Três instrumentos são aplicados na pesquisa (guia de revisão bibliográfica, redação e questionário). Os resultados mostram a necessidade de continuar diagnosticando, na prática, a eficácia do modelo de educação a distância e continuar desenvolvendo as competências-chave para enfrentar metodologicamente o ensino nas condições do COVID-19. Propõe-se ampliar a pesquisa a um universo maior de docentes para diagnosticar e influenciar nas habilidades que ainda não foram suficientemente desenvolvidas.

Palavras-chave: competências de ensino; ensino; aprendizagem; COVID-19; educação.

Competencies in university teaching staff derived from the impact of COVID-19

Abstract. The COVID-19 pandemic has put the world's Higher Education Institutions to the test. Partial or total closure of classrooms, curricular adaptations and the halted teaching-learning process are some of the negative consequences of the pandemic. The objective of this article is to define the competencies developed by the university teacher to methodologically face teaching in conditions of COVID-19. 12 teachers from the "Marta Abreu" Central University of Las Villas participate in the research. The research, with a mixed, non-experimental and descriptive approach, is carried out in two stages. The first determines the key competencies of the university teacher to methodologically face teaching in conditions of COVID-19. The second describes the current state of these key competencies in university teaching staff. Three instruments are applied in the research (guide for bibliographic review, composition and questionnaire). The results show the need to continue diagnosing, in practice, the effectiveness of the distance education model and to continue developing the key competencies to methodologically face teaching in COVID-19 conditions. It is proposed to extend the research to a larger universe of teachers to diagnose and influence the skills that have not yet been sufficiently developed.

Keywords: teaching skills; teaching; learning; COVID-19; education. 


\section{Introducción}

El docente universitario actual realiza su labor en una época marcada por el desarrollo tecnológico, las crisis económicas, las pandemias y el cambio climático. El complejo contexto donde desarrolla su labor, junto a la enorme rapidez con la que se multiplican los conocimientos, influye en la transformación de escenarios tradicionales de la educación. Además, exige mayor preparación del docente (Krishnamurthy, 2020; Yao \& Guo, 2018).

En la actualidad, la pandemia de la COVID-19 ha impactado los conocimientos, habilidades y valores del docente universitario. El aislamiento social, los encierros y la ausencia del contacto personal con los estudiantes conllevan a evolucionar, en ocasiones, aceleradamente hacia un escenario de enseñanza-aprendizaje virtual.

El 24 de marzo de 2020 las Instituciones de Educación Superior cubanas (IES) cierran sus puertas (Granma, 2020). La situación epidemiológica desatada por la COVID-19 obliga a más de 241.000 estudiantes a interrumpir sus estudios. La didáctica de la educación superior sufre cambios.

El docente universitario se ve obligado a modificar el diseño curricular para poder planificar el proceso de enseñanza-aprendizaje. Propósitos, contenidos, empleo de tecnologías, distribución temporal (orden) y evaluaciones, tienen que ser adaptadas a las nuevas condiciones de la enseñanza universitaria (Aguilera-Hermida, 2020). Además, es necesario confeccionar materiales bibliográficos (libros electrónicos, webquest, edublogs), aulas virtuales y actividades interactivas para provocar los cambios necesarios en los estudiantes respecto a la asimilación del conocimiento.

Las nuevas condiciones en las que se desarrolla el proceso de enseñanzaaprendizaje universitario requirió una formación continua y acelerada en el profesorado; a fin de satisfacer las necesidades educativas de los estudiantes. Por otra parte, propició un cambio sustantivo del modelo de enseñanza tradicional. La práctica educativa comenzó a exigir, como nunca, aplicar diferentes pedagogías para obtener el máximo aprovechamiento en el proceso de enseñanza-aprendizaje. El objetivo del artículo es definir cuáles son las competencias desarrolladas por el docente universitario para enfrentar metodológicamente la docencia en condiciones de la COVID-19.

\section{Métodos y materiales}

La investigación realizada presenta un enfoque mixto, no experimental y con carácter descriptivo. Se estructura para ser aplicada en dos etapas. La primera etapa centra su atención en determinar, a través de la búsqueda bibliográfica, las competencias claves del docente universitario para enfrentar metodológicamente la docencia en condiciones de la COVID-19. En la segunda etapa se describe el estado actual de las competencias claves para enfrentar metodológicamente la docencia en condiciones de la COVID-19 en el profesorado universitario.

\subsection{Participantes}

La muestra para la investigación está compuesta por 12 docentes de los Departamentos de Español-Literatura, Educación Especial y el Departamento de Preparación y Superación de Cuadros de la Universidad Central "Marta Abreu" de Las 
Villas. El 42\% de los participantes son del sexo masculino. El 58\% restante, son del sexo femenino. El participante de más experiencia, frente a las aulas, tiene 64 años de edad. El más joven, cuenta con 32 años.

\subsection{Instrumento}

En la investigación se aplican tres instrumentos. El primer instrumento es la Guía para la revisión bibliográfica. El objetivo es determinar cuáles son las competencias que debe reunir el profesorado universitario para enfrentar metodológicamente la docencia en condiciones de COVID-19. El segundo instrumento aplicado es la Composición (Martínez-Angulo et al., 2013). El objetivo que se persigue es obtener información sobre el mundo interno de los sujetos respecto a las competencias claves determinadas para enfrentar metodológicamente la docencia en condiciones de COVID-19. EI tercer instrumento utilizado es el Cuestionario. El objetivo de este instrumento consiste en determinar cómo ha sido el desarrollo de las competencias claves para enfrentar metodológicamente la docencia en condiciones de la COVID-19

El valor alfa de Cronbach obtenido $(\alpha=0,950)$ muestra la excelente fiabilidad del cuestionario para conocer la opinión de los participantes sobre las competencias claves determinadas.

\subsection{Procedimiento y análisis}

Para la ejecución del primer instrumento se siguen los pasos planteados por la Facultad de Ciencias Médicas "10 de octubre" (2018). Los investigadores recopilan bibliografía en las bases de datos especializadas. Posteriormente, evalúan y seleccionan la bibliografía acorde a la relevancia y calidad de estas. Un tercer paso conlleva a procesar la información obtenida y llegar a conclusiones, acorde al objetivo planteado en el instrumento. El segundo instrumento se aplica a los 12 participantes. A partir del título ¿Qué competencias necesito para impartir docencia durante la pandemia?, los sujetos redactan las composiciones. De este resultado se conforma un sistema de categorías inductivo (ver Tabla 1), a través del cual se analizan las respuestas ofrecidas. Para el tercer instrumento se analizan los datos a través del software SPSS versión 23. Los investigadores realizan un análisis descriptivo donde exponen el estado actual de las competencias claves para enfrentar metodológicamente la docencia en condiciones de la COVID-19 en los participantes. El análisis descriptivo considera la media y la desviación típica de los ítems.

Tabla 1. Sistema de categorías para el análisis de las composiciones

\begin{tabular}{ll}
\hline Categorías & Indicadores \\
Conocimientos & $\begin{array}{l}\text { El sujeto expresa saberes reflejados para enfrentar metodológicamente la } \\
\text { docencia en condiciones de la COVID-19. }\end{array}$ \\
Habilidades & $\begin{array}{l}\text { El sujeto demuestra experiencias donde pone de manifiesto destrezas para } \\
\text { enfrentar metodológicamente la docencia en condiciones de la COVID-19. }\end{array}$ \\
Actitudes & El sujeto expone modos de actuación para enfrentar metodológicamente \\
& la docencia en condiciones de la COVID-19.
\end{tabular}




\section{Resultados}

La primera etapa de la investigación arroja, en los últimos tres años, un total de 2500 investigaciones que, de alguna manera, están relacionadas con competencias claves del docente universitario para enfrentar metodológicamente la docencia en condiciones de la COVID-19. Estas investigaciones se ubican en bases de datos especializadas [Google Scholar, Education Resources Information Center (ERIC)], así como, en repositorios institucionales. La revisión bibliográfica muestra como resultado que las competencias son unidades integradas y complejas. Las competencias de un docente no se reducen solo a las acciones ejecutadas por el individuo en el desempeño de tareas concretas; sino que incluyen diferentes componentes psicológicos o personológicos interrelacionados (Aguilera-Hermida, 2020).

Las competencias de un docente actual para enfrentar metodológicamente la docencia en condiciones de la COVID-19 no pueden determinarse pensando solo en el aula tradicional (situada en un espacio físico; entre muros). Las clases se imparten en variados escenarios. El docente debe saber cómo reorganizar el contenido específico, reajustar la planificación, organizar actividades individuales o grupales, motivar e involucrar a los estudiantes; fomentar el aprendizaje colaborativo y tener presente la salud mental y el bienestar del estudiantado desde la distancia.

El análisis realizado por los investigadores y teniendo presente criterios aportados por Dwivedi et al. (2020); Jiménez-Guerra y Ruiz-Gonzáles (2021); Lealh-Filho (2021); Ruiz-Cuellar (2020) y Tzivinikou et al. (2021) permiten determinar las competencias claves del docente universitario para enfrentar metodológicamente la docencia en condiciones de la COVID-19 (ver Figura 1).

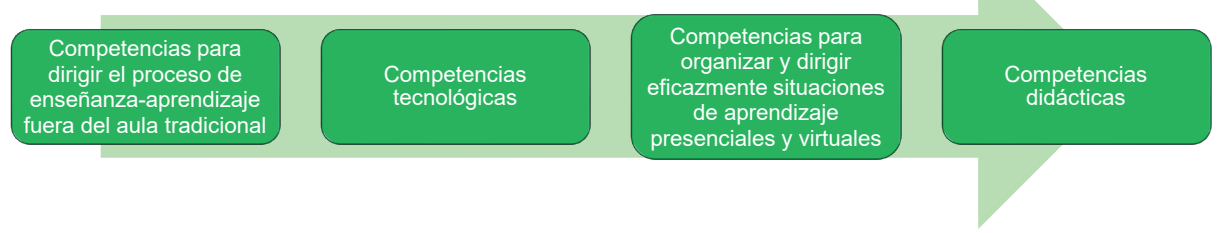

Figura 1. Competencias claves del docente universitario emanadas de la revisión bibliográfica.

Fuente: elaboración propia

La aplicación de los instrumentos Composición y Cuestionario permiten, en la segunda etapa, describir el estado actual de las competencias claves para enfrentar metodológicamente la docencia en condiciones de la COVID-19 de la población objeto de estudio. Teniendo presente el sistema de categorías previamente elaborado (ver Tabla 1), se realiza un análisis de las respuestas ofrecidas por los participantes en la composición. El resultado se organiza según las categorías determinadas.

\subsection{Conocimientos}

Los sujetos muestran saberes para proponer situaciones problémicas, ajustándolas a las potencialidades de cada estudiante. Los conocimientos para dirigir el proceso de enseñanza-aprendizaje fuera del aula tradicional se han incrementado. 
En las respuestas de los sujetos se reconoce saberes sobre el sistema operativo empleado; para la utilización pedagógica de las herramientas de comunicación (correo electrónico, mensajería instantánea) y para emplear recursos tecnológicos (app, libros digitales) en sus clases.

Aprendíde manera autodidacta y ayudada desde la distancia por mis compañeros a planificar y organizar mi docencia desde el aula virtual. Considero que he sido útil a la sociedad, a pesar de las restricciones. Considero que he aprendido a manejar escenarios virtuales para ofrecer atención académica y psicológica a mis estudiantes (Sujeto 5).

\subsection{Habilidades}

Las respuestas de los sujetos muestran desarrollo de habilidades tecnológicas y didácticas para organizar situaciones de aprendizaje desde la distancia. Dominan condiciones básicas para emplear la plataforma Moodle. Además, reconocen contar en sus clases con un mayor empleo del Word y el Power Point. Por otra parte, han incrementado las destrezas para la búsqueda, en sitios especializados, de artículos científicos dirigidos a el tratamiento de los contenidos. En general, han adquirido destrezas para organizar, planificar, ejecutar y evaluar el aprendizaje de sus estudiantes apoyados por las tecnologías.

La pandemia nos enseñó a vernos útiles desde la casa y a trabajar por el bien de la Educación cubana. Los resultados alcanzados nos han hecho comprender que fuera del aula, apoyados por el Moodle, también se puede impartir clases. La pandemia nos influyó positivamente en el manejo de herramientas tecnológicas que anteriormente no empleábamos (Sujeto 11).

\subsection{Actitudes}

La mayoría de los participantes muestra una actitud positiva para acompañar el aprendizaje de los estudiantes fuera del aula tradicional. Estos sujetos han empleado las tecnologías para ofrecer continuidad al proceso de enseñanza-aprendizaje; detenido producto a la pandemia. La mayoría de los sujetos demuestra ser capaz de impartir contenidos apoyados por el aula virtual y el WhatsApp. En los escritos manifiestan voluntad para continuar utilizando recursos tecnológicos en sus clases.

Estos meses han sido duros para mí. El aislamiento social ha constituido una escuela que ha formado a esta docente de 47 años en el verdadero empleo de las tecnologías. Además, nos ha hecho valorar más al ser humano y la vida en general. Me he erigido como mejor docente, sintiendo la necesidad de luchar para poder trabajar en las nuevas condiciones y solucionar problemas con criterios tecnológicos (Sujeto 1).

Los resultados alcanzados en el instrumento Cuestionario se muestran en la Tabla 2. La dimensión Conocimientos alcanza una media alta $(M=3.63)$ reflejando el incremento de los saberes para enfrentar metodológicamente la docencia en condiciones de COVID-19. La desviación típica de los ítems es inferior a 1 en el 100\% de los casos. El 21\% de los ítems no muestra desviación típica alguna. Los conocimientos que el profesorado estima poseer, en menor medida, son el ítem 4a $(M=3.33 ; \mathrm{DT}=.492)$, el 4c $(M=3.42 ; D T=.515)$, el 3e $(M=3.42 ; D T=.793)$ y el ítem $2(M=3.48 ; D T=.793)$. Los mayores conocimientos se observan en el ítem 3d (M=4.00; $D T=.000)$, el 4b (M= 4.00; $\mathrm{DT}=.000)$ y el $4 \mathrm{~d}(\mathrm{M}=4.00 ; \mathrm{DT}=.000)$. 
La dimensión Habilidades alcanza también una media alta $(M=3.44)$. El resultado constata el desarrollo de destrezas para enfrentar metodológicamente la docencia en condiciones de COVID-19. Similar a los resultados obtenidos en la dimensión Conocimientos, ninguno de los ítems alcanza una desviación típica superior a 1. Las destrezas que los sujetos creen haber desarrollado en menor medida se muestran en el ítem $4 a$ $(\mathrm{M}=3.33 ; \mathrm{DT}=.492)$; mientras que, las destrezas que alcanzaron el mayor desarrollo se observan en el ítem 4b ( $M=4.00$; DT=. 000) y el ítem 4e $(M=3.58 ; D T=.515)$.

La dimensión Actitud alcanza la media más alta de las tres dimensiones $(\mathrm{M}=$ 4.00). El 100\% de los ítems pertenecientes a la dimensión carece de desviación típica alguna (DT = .000). La totalidad de los sujetos ha demostrado fortalecer la actitud para enfrentar metodológicamente la docencia en condiciones de la COVID-19.

Tabla 2. Análisis descriptivo de los ítems presentes en el Cuestionario

\begin{tabular}{lccc}
\hline \multicolumn{1}{c}{ Ítem } & M & DT \\
1. Conocimientos didácticos para asegurar la enseñanza de tu disciplina & 3.58 & .515 \\
2. Conocimientos requeridos para la gestión de contenidos digitales & 3.48 & .793 \\
3. Conocimientos para la utilización de las TIC en el desarrollo del aprendizaje & & \\
3 a. Manejo de la plataforma educativa Moodle & 3.08 & .669 \\
3 b. Sistemas operativo Windows & 3.75 & .452 \\
3 c. Sistemas operativo Linux & 3.58 & .515 \\
3 d. Programa de presentación Power Point & 4.00 & .000 \\
3 e. Diseño de materiales didácticos para el aprendizaje on-line & 3.42 & .793 \\
3 f. Empleo de softwares educativos desde la PC & 3.83 & .389 \\
3g. Uso de recursos electrónicos, páginas web, bases de datosy catálogos en línea & 3.75 & .452
\end{tabular}

4. Conocimientos para atender las diferencias individuales, el desarrollo emocional y la salud mental
4 a. Atención personalizada on-line
$3.33 \quad .492$
4 b. Atención al temor e inseguridad
$4.00 \quad .000$
4 c. Atención al desarrollo socio-emocional del estudiante
$3.42 \quad .515$
4 d. Elevación de la motivación e interés
$4.00 \quad .000$
4 e. Atención a los diferentes problemas que genera la pandemia en la salud mental
$3.58 \quad .515$

5. Habilidades en la Educación superior en tiempos de COVID-19

5 a. Preparar los materiales de las clases on-line $\quad 3.67 \quad .492$

5 b. Evaluar a los estudiantes on-line $\quad 3.58 \quad .515$

$\begin{array}{lll}5 \text { c. Utilizar recursos de Internet para impartir tus clases } & 3.50 & .674\end{array}$

$\begin{array}{llll}5 \text { d. Desarrollo de la enseñanza hibrida } & 3.17 & .718\end{array}$

5e.Utilización de las TIC al servicio del aprendizajey de laadquisición deconocimiento $\quad \begin{array}{lll}3.33 & .778\end{array}$

$5 \mathrm{f}$. Atención a las diferencias individuales a distancia $\quad 3.42 \quad .515$

6. Actitud en la Educación superior en tiempos de COVID-19

$\begin{array}{llll}6 \text { a. La enseñanza universitaria puede continuar en tiempos de COVID-19 } & 4.00 & .000\end{array}$

6 b. La enseñanza hibrida puede ser aplicada a la Educación superior $\quad 4.00 \quad .000$

$6 \mathrm{c}$. Las tecnologías son viables para desarrollar el proceso enseñanza-aprendizaje $\quad \begin{array}{lll}4.00 & .000\end{array}$

6 d. Las tecnologías facilitan la atención a las diferencias individuales $\quad \begin{array}{lll}4.00 & .000\end{array}$

Fuente: elaboración propia. 


\section{Discusión}

El análisis de los datos y la información obtenida evidencian un alto desarrollo de competencias adquiridas para enfrentar metodológicamente la docencia en condiciones de la COVID-19. A pesar de los sujetos, inicialmente, no contar con la preparación necesaria para impartir clases fuera del escenario tradicional; son capaces, con la ayuda de sus compañeros, la preparación a distancia recibida desde la universidad y la autopreparación, de promover transformaciones en sus conocimientos, habilidades y actitudes hacia la educación a distancia. Este primer resultado contrasta con lo expuesto por Sierralta-Pinedo (2021) en su investigación. El estudio realizado por la investigadora demuestra que, a un año de pandemia, docentes de la Institución Educativa CECAT "Marcial Acharán” tienen escasas competencias digitales y pedagógicas para impartir clases desde la virtualidad.

Por otra parte, los resultados alcanzados en la investigación están acordes con las competencias docentes declaradas por Garzón-Daza (2021) en su estudio. Este investigador estima que el docente, producto a la situación del COVID, ha tenido que transformar su forma de enseñar, al tener que integrar lo físico con lo virtual. Precisamente, este resultado es observado en la investigación. Los 12 sujetos han desarrollado una alta capacidad de adaptación e innovación producto a la incorporación de las tecnologías al proceso de enseñanza-aprendizaje. Además, realizar esta investigación cumple con una de las directrices planteadas por Vite-Ceballos et al. (2020):

Cada IES debe establecer una hoja de ruta que identifique qué aplicaciones TIC, TAC, TEP requiere hacer uso, tomando en cuenta la naturaleza de cada asignatura para de esa manera establecer las áreas de desarrollo a través de procesos de capacitación. (p. 183)

\section{Conclusiones}

La investigación ejecutada permitió realizar un acercamiento a los principales impactos que ha provocado la pandemia en la preparación del profesorado universitario a partir de las competencias requeridas para su enfrentamiento

Un primer impacto está relacionado con la necesidad de continuar diagnosticando, en la práctica, la efectividad del modelo de educación a distancia. Este modelo se desarrolla a través de diferentes tecnologías. Además, debe tener presente el fomento del aprendizaje colaborativo, la deserción escolar y los estados socio-emocionales de los estudiantes (Garrido et al., 2021; Rieble-Aubourg \& Viteri, 2020). No todos los docentes presentan las competencias necesarias para incorporar el modelo de educación a distancia a su accionar diario.

Un segundo impacto se puede circunscribir en la necesidad de continuar desarrollando en el profesorado universitario las competencias claves para enfrentar metodológicamente la docencia en condiciones de la COVID-19. La pandemia aún no finaliza. El docente debe continuar preparándose. Además, se pueden aprovechar los aprendizajes que va dejando la COVID-19 y seguir avanzando en la capacitación para enfrentar otras pandemias; desarrollando al máximo estas y otras competencias clave que le permitan favorecer el proceso de enseñanza-aprendizaje integrando tecnologías, conocimientos y didácticas. 
Para finalizar, se propone ampliar la investigación a un universo mayor de docentes. Es una necesidad diagnosticar cuáles son las competencias desarrolladas por el profesorado universitario para enfrentar metodológicamente la docencia en condiciones de la COVID-19; e influir en aquellas que aún no han sido lo suficientemente desarrolladas.

\section{Referencias}

Aguilera-Hermida, P. (2020). College students' use and acceptance of emergency online learning due to COVID-19. International Journal of Educational Research, 366-374. https://doi. org/10.1016/j.ijedro.2020.100011.

Dwivedi, Y., Hughes, L., Coombs, C., Constantiou, I., Y. Duan, Y. \& Edwards, J. (2020). Impact of COVID-19 pandemic on information management research and practice: Transforming education, work and life. International Journal of Information Management, 55. https://doi. org/10.1016/j.ijinfomgt.2020.102211

Facultad de Ciencias Médicas «10 de octubre» (2018). Guía para elaborar una Revisión bibliográfica. Recuperado de https://instituciones.sld.cu.

Garrido, C., Garrido, A., Álvarez, P. y Vargas, S. (2021). Competencias digitales para la industria 4.0. Efectividad del proceso de virtualización de un laboratorio de Metrología en la carrera de Ingeniería Mecánica. EDUTEC. Revista Electrónica de Tecnología Educativa, 76, 197211. https://doi.org/10.21556/edutec.2021.76.1923.

Garzón-Daza, C. (2021). Las competencias docentes en el siglo XXI de cara a la virtualidad de la Educación con ocasión del Covid-19 . Revista Boletín REDIPE,10(5), 177-188. https:// reista.redipe.org/index.php/1/article/download/12995/1203.

Granma (24 de marzo de 2021). Nuevas medidas para enfrentar a la COVID-19. Recuperado de https://www.granma.cu/cuba-covid-19/2021-03-24.

Jiménez-Guerra, Y. y Ruiz-Gonzáles, M. D. (2021). Reflexiones sobre los desafíos que enfrenta la Educación superior en tiempos de covid-19. Economía y Desarrollo, 165(1), 1-15. https:// bit.ly/3gU9Vow.

Krishnamurthy, S. (2020). The future of business education: A commentary in the shadow of the Covid-19 pandemic. Journal of Business Research, 117, 1-5. https://doi.org/10.1016/j. jbusres.2020.05.034.

Leal-Filho, W. (2021). Covid-19, Sustainable development and higher education: towards a recovery path. Journal of Sustainability in Higher Education, 138-141. http://doi.org/10.1108/ IJSHE-10-2020-0364.

Martínez-Angulo, M. R., Álvarez-Dorta, L., Yaque-Villegas, E. L., Hernández-Pardo, I. y RodríguezDíaz, Z. (2013). Manual de técnicas de exploración piscólógicas. Pueblo y Educación.

Rieble-Aubourg, S. y Viteri, A. (2020). Hablemos de Política Educativa en América Latina y el Caribe. División de Educación y desarrollo social del Banco Interamericano de Desarrollo.

Ruiz-Cuellar, G. (2020). Covid-19: pensar la educación en un escenario inédito. Revista Mexicana de Investigación Educativa, 25(85), 229-237. https://bit.ly/3rQGlah.

Sierralta-Pinedo, S. (2021). Competencias digitales en tiempos de COVID-19, reto para los maestros de la institución educativa CECAT "Marcial Acharán". Revista Mendive, 19(3), 755-763. https://bit.ly/3oOhPUY.

Tzivinikou, S., Garifalia, C. \& Kagkara, D. (2021). Distance education attitudes (DEAS) during COVID 19 crisis: Factor structure, reliability and construct validity of the brief Dea scale in greekspeaking SEND teachers. Technology, Knowledge and Learning, 26(3), 461-479. https:// doi.org/10.1007/s10758-020-09483-1. 
Vite-Cevallos, H., Carvajal-Romero, H., Samaniego-Ocampo, R. y Prado-Ortega, M. (2020). Competencias virtuales de los docentes frente al reto de COVID-19 en instituciones de Educación Superior en Ecuador. Revista Conrado, 16(1), 178-183. https://bit.ly/3rQm8RG

Yao, J.-X. \& Guo, Y.-Y. (2018). Core competences and scientific literacy: the recent reform of the school science curriculum in China. International Journal of Science Education, 40(3), 1913-1933. https://doi.org/10.1080/09500693.2018.1514544.

Cómo citar en APA:

Martínez, C., Martínez, R. y Vázquez, D. (2022). Competencias en el profesorado universitario derivadas del impacto de la COVID-19. Revista Iberoamericana de Educación, 88(1), 101-109. https://doi.org/10.35362/rie8814823 